\section{Ultrasonic appearance probably indicative of imminent fetal death}

One of the difficulties in recognising fetal death by ultrasonic scanning in early pregnancy is that the negative finding of no fetal heart movement must always be less convincing than a positive finding. Furthermore, Robinson ${ }^{1}$ found only one instance of irregularity of the fetal heart in ten cases scanned before their pregnancies became missed abortions, and he concluded that it was not yet possible to predict fetal death by sonar. I report an ultrasonic appearance that I have noted on at least 14 occasions always in association with subsequent fetal death.

\section{Case reports}

Case 1-A 33-year-old housewife had had one previous pregnancy $2 \frac{1}{2}$ years earlier. She was first seen in the current pregnancy 12 weeks after her last menstrual period. Two weeks later she had a slight brownish vaginal discharge, and on clinical examination her uterus was felt to be small for dates. She was therefore referred for ultrasonic scanning. The scan was performed 14 weeks after her last period using a Diasonagraph NE 4102 (Nuclear Enterprises, Edinburgh) with a sound frequency of $2.5 \mathrm{MHz}$ and pulse repetition frequency of $600 \mathrm{~Hz}$. Fetal crown rump length was only $50 \mathrm{~mm}$, and a fine vibration was noted originating from the region of the fetus (figure). The vibration was intermittent and variable in rate but about $1200-1500 \mathrm{~Hz}$. On repeat scan three days later no fetal heart movement or vibration could be detected. An assay of the serum concentration of the $\beta$ subunit of human chorionic gonadotrophin gave an inappropriately low value and it was decided to evacuate the uterus surgically. This was carried out four days later, the products being histologically compatible with a missed abortion.

Case 2-A 27-year-old housewife was referred for termination of pregnancy. An ultrasonic scan (details as in case 1) carried out seven weeks after her last menstrual period showed an intrauterine gestational sac containing a fetus with a crown rump length of $14.7 \mathrm{~mm}$. The fetal heart movement was easily detected and had a rate of 150 beats/min. Termination of the pregnancy was agreed and, with the patient's consent, it was decided to perform this by the intravaginal administration of a pessary containing an ester of 15-methylprostaglandin $\mathrm{F} 2 \alpha^{2}{ }^{2}$ As part of the trial of the abortion method further ultrasonic scans were performed seven hours after insertion of the pessary and two days later. On the earlier of these scans no fetal heart movement could be detected within the partially fragmented gestational sac; but on the later scan very slow, irregular movements of fetal heart type were seen in the region of the fetus, and superimposed upon these were intermittent rapid vibrations, the rate of which was about $1500 \mathrm{~Hz}$.

\section{Comment}

In both cases described the fetal echoes were too small to allow further identification of the source of these vibrations, but when the phenomenon has been observed in larger fetuses it appears to originate

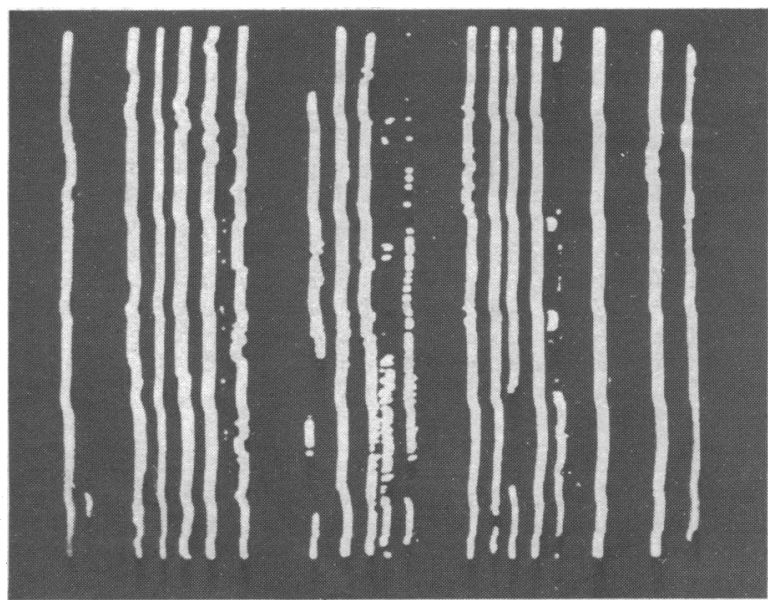

Time-position scan (time base movement $25 \mathrm{~mm} /$ minute) showing rapid vibrations in central echoes. from the thoracic area in the position where the heart movement might be expected. The rate of the vibration is, of course, much faster than the heart rate but it does not appear to be an artefact since it is localised to the region of the fetal echoes and is intermittent, usually, lasting for only one or two seconds at a time. Furthermore, I havew never observed this appearance in pregnancies which subsequentlyz progressed normally.

The mechanism of production of this phenomenon is unknown but possibly the biochemical changes associated with imminent fetal ${ }^{-}$ death give rise to myocardial fibrillation and it is the resulting move- $\overrightarrow{\vec{n}}$ ments which are detected. I should be most interested to hear of any similar observations and to learn of any alternative hypothesis, either physiological or electronic, to account for this appearance.

I thank the consultant staff of Charing Cross Hospital for referring their patients for ultrasonic scanning, and in particular $\mathrm{Mr}$ M G Elder (now Professor Elder) for allowing me to include details of the two illustrative cases.

${ }^{1}$ Robinson, H P, British fournal of Obstetrics and Gynaecology, 1975, 82, $\overrightarrow{\vec{\omega}}$ 855 and 856.

2 Dutt, T P, Blair, M, and Elder, M G, American fournal of Obstetrics and Gynecology. In press.

(Accepted 19 May 1978)

Charing Cross Hospital Medical School, London W6 7DQ

T P DUTT, MRCOG, lecturer and honorary senior registrar in obstetrics and $\infty$ gynaecology

\section{Paracetamol poisoning in children}

The number of patients admitted to hospital because of paracetamol poisoning has increased since $1968,{ }^{1}$ and in 1975 there were 105 deaths due to paracetamol. ${ }^{2}$ A further 107 deaths occurred due toㅇํ paracetamol in combination with other drugs. ${ }^{2}$ There are no official statistics available for the incidence and severity of paracetamole poisoning in children, and we therefore reviewed information collected $\overrightarrow{\hat{\sigma}}$ by the National Poisons Information Service (NPIS) over a three-3 year period.

\section{Patients, methods, and results}

In 1975-7 the NPIS received 3139 inquiries about paracetamol poisoning, and 580 of these concerned children under the age of 13 years. Questionnaires 3 were sent in each case to the relevant doctor asking for details of the age and sex of the patient, the brand of paracetamol and the quantity ingested, the results of plasma paracetamol estimations and liver function tests, the treatment given, and the outcome. Replies to 116 questionnaires were음 received.

Analysis of the age and sex distribution (see table) showed that the patients $N$ fell into two main groups ( 1 to 4 years and 11 to 13 years) with different $D$ sex ratios. Of the 86 patients aged 1 to 4 years, 24 ingested paracetamolo tablets, 34 ingested paracetamol elixirs, and 22 ingested Distalgesic tablets (paracetamol $325 \mathrm{mg}$ and dextropropoxyphene $32.5 \mathrm{mg}$ ); the figures for the $\mathrm{N}$ 27 patients aged 11 to 13 years were 16,0 , and 5 respectively (brand not $N$ stated in 12 cases). In 49 of the 86 patients aged 1 to 4 years the quantity of paracetamol taken was known, and in only eight cases did it exceed $5 \mathrm{~g}$. 우 Among 11- to 12-year-olds, however, the amount of paracetamol wasco known in 23 cases, and in 18 of these the amount exceeded $5 \mathrm{~g}$.

Paracetamol taken alone caused vomiting and drowsiness in 15 patients. Of the 221 - to 4-year-olds who took Distalgesic tablets, 18 had no symptoms, one suffered abdominal pain, one vomited (Distalgesic and iron tablets), one was drowsy (Distalgesic and prochlorperazine), and a 4-year-old girl who ingested 20 to 25 Distalgesic tablets became unconscious. Five children aged 11 to 13 years took Distalgesic tablets: three were asymptomatic and $\mathbb{\Phi}$ two became unconscious (one took 30 tablets and the other took a barbiturate $\varrho$ as well).

Plasma paracetamol concentrations were determined in 52 patients, and 0 in 15 cases paracetamol was not detected. In three cases the paracetamolo concentrations fell above a line joining $200 \mathrm{mg} / 1$ at 4 hours and $80 \mathrm{mg} / 1$ at 12 hours after ingestion when plotted on a semilog paracetamol-time scale. In only one of these patients did the maximum plasma aspartate aminotransferase concentration (SGOT) exceed $250 \mathrm{IU} / 1$ : a 13-year-old girl ingested 15 - to $20-\mathrm{g}$ paracetamol 12 hours before hospital admission. The initial paracetamol concentration was $221 \mathrm{mg} / \mathrm{l}$ and she was given $5 \mathrm{~g}$ of methionine over the following 21 hours. Hepatic encephalopathy developed 